\title{
Bioconductor caAffy Average Difference Probe Summary Method
}

National Cancer Institute

\section{Source}

National Cancer Institute. Bioconductor caAffy Average Difference Probe Summary

Method. NCI Thesaurus. Code C64222.

Summarizes probe sets by computing the average difference between all PM and MM probes in a probe set. Defined in the documents available at

http://bioconductor.org/packages/1.9/bioc/html/affy.html 